\title{
On Infinite Series and with Their Some Applications to Euler's Summation Formula
}

\author{
Abdel Radi Abdel Rahman Abdel Gadir Abdel Rahman, Neama Yahia Mohammed, \\ Subhi Abdalazim Aljily, and Nidal Elamen Mohammed Ali
}

\begin{abstract}
Infinite series is still used in engineering, mathematical and physical sciences.In the modern area a great progress is made in the sciences of calculus in addition to what was accompanied by advances in infinite series and their some applications. The aims of this paper is to develop and introduces the infinite series and their some applications to Euler's summation, also we show and explain how to apply the infinite series in Euler's summation. We followed the induction mathematical method and found that : The relationship through the Euler's summation focused on the physical link questioner to make the study as an application for Euler's summation so it can be the beginning of advanced study in concept of the infinite series and their some applications to Euler's summation.
\end{abstract}

Index Terms - Applications, Euler's Summation Formula, Infinite Series.

\section{INTRODUCTION}

In this study, we deal with an infinite series and some applications to Euler's summation and asymptotic expansions. Firstly, we spoke about the sequences, then we dealt with the derivatives, after that we explained different concepts which related to infinite series, finally we discuss the vectors and applied Euler's summation formula and asymptotic expansions to infinite series.

\section{THE INFINITE SERIES}

\section{A. Definition}

If $\left\{a_{n}\right\}_{k}^{\infty}$ is an infinite sequence of real numbers, the symbol $\sum_{n=k}^{\infty} a_{n}$ is an infinite series, and $a_{n}$ is the $n$th term of the series. We say that $\sum_{n=k}^{\infty} a_{n}$ is converges to the sum $A$, and write $\sum_{n=k}^{\infty} a_{n}=A[4]$ if the sequence $\left\{A_{n}\right\}_{k}^{\infty}$ defined by:

$$
A_{n}=a_{K}+a_{K+1} \ldots \ldots \ldots+a_{n}, n \geq k \text {; }
$$

converges to $A$.

The finite sum $A_{n}$ is the $n$th partial sum of $\sum_{n=k}^{\infty} a_{n}$. If $\left\{A_{n}\right\}_{k}^{\infty}$ diverges, we say that $\left\{A_{n}\right\}_{k}^{\infty}$ diverges; in particular, if $\lim _{n \rightarrow \infty} A_{n}=\infty$ or $-\infty$, we say that $\sum_{n=k}^{\infty} a_{n}=\infty$ diverges to $\infty$ or $-\infty$, and write $\sum_{n=k}^{\infty} a_{n}=\infty$ or $\sum_{n=k}^{\infty} a_{n}=-\infty$.

A divergent infinite series that does not diverge to $\pm \infty$ is said to oscillate or be oscillatory.

We usually refer to infinite series more briefly as series. [17].

\section{Published on July 05, 2021}

Abdel Radi Abdel Rahman Abdel Gadir Abdel Rahman, Associate Professor Department of Mathematics, Faculty of Education, Omdurman Islamic University, Omdurman, Sudan.

(e-mail: dibdelradi78@gmail.com)

\section{B. Example}

Consider the series $\sum_{n=0}^{\infty} r^{n},-1<r<1$.

Here $a_{n}=r^{n} .(n>0)$ and

$$
A_{n}=1+r+r^{2}+\ldots \ldots+r^{n}=\frac{1-r^{n+1}}{1-r}
$$

which converges to $\frac{1}{1-r}$ as $n \rightarrow \infty$; thus, we write:

$$
\sum_{n=0}^{\infty} r_{n}=\frac{1}{1-r},-1<r<1 \text { if }|r|>1
$$

then (1) is still valid, but $\sum_{n=0}^{\infty} r_{n}$ Diverges, if $r>1$, then

$$
\sum_{n=0}^{\infty} r_{n}=\infty
$$

If $r<1, \sum_{n=0}^{\infty} r_{n}$ oscillates, since its partial sums alternate in sign and their magnitudes become arbitrarily large for large $n$. If $r=-1$, then $A_{2 m+1}=0$ and $A_{2 m}=1$ for $m \geq$ 0 , while if $r=1, A_{n}=n+1$; in both cases the series diverges, and (2) holds or $r=1$ [1].

The series $\sum_{n=0}^{\infty} r_{n}$ is called the geometric series with ratio. It occurs in many applications. An infinite series can be viewed as a generalization of a finite sum

$$
A=\sum_{n=k}^{N} a_{n}=a_{K}+a_{K+1} \ldots \ldots \ldots+a_{N}
$$

By thinking of the finite sequence $\left\{a_{K}, a_{K+1}, \ldots, a_{N}\right\}$ as being extended to an infinite sequence $\left\{a_{n}\right\}_{k}^{\infty}$, with $a_{n}=0$ for $n>N$. Then the partial sums of $\sum_{n=k}^{\infty} a_{n}$ are:

$$
A_{n}=a_{K}+a_{K+1} \ldots \ldots \ldots+a_{n}, k \leq n<N,
$$

And

$$
A_{n}=A, n \geq N[6]
$$

That is, the terms of $\left\{a_{n}\right\}_{k}^{\infty}$ equal the finite $\operatorname{sum} A$ for $n \geq$ $k$. Therefore, $\lim _{n \rightarrow \infty} A_{n}=A$.

\section{Theorem}

Let $\sum_{n=k}^{\infty} a_{n}=A$ and $\sum_{n=k}^{\infty} b_{n}=B$ where $A$ and $B$ are finites: Then $\sum_{n=k}^{\infty} c a_{n}=c A, c$ is a constant.

Neama Yahia Mohammed, Assistant Professor Department of Mathematics, College of Science, Tabuk University, Saudi Arabia.

Subhi Abdalazim Aljily, Assistant Professor Department of Mathematics, Faculty of Education, University of AL Butana, Sudan.

Nidal Elamen Mohammed Ali, Department of Mathematics, Faculty of Education, Omdurman Islamic University, Omdurman, Sudan. 
$\sum_{n=k}^{\infty}\left(a_{n}+b_{n}\right)=A+B$ and $\sum_{n=k}^{\infty}\left(a_{n}-b_{n}\right)=A-B$ [11].

These relations also hold if one or both of $A$ and $B$ is infinite, provided that the right sides are not indeterminate: Dropping finitely many terms from a series does not alter convergence or divergence, although it does change the sum of a convergent series if the terms dropped have a nonzero sum. For example, suppose that we drop the first $k$ terms of a series $\sum_{n=0}^{\infty} a_{n}$ an, and consider the new series $\sum_{n=k}^{\infty} a_{n}$. Denote the partial sums of the two series by:

$$
A_{n}=a_{0}+a_{1}+\cdots \ldots+a_{n}, n \geq 0
$$

And

$$
A_{n}^{\prime}=a_{K}+a_{K+1} \ldots \ldots \ldots+a_{n}, n \geq k .
$$

Since

$$
A_{n}=\left(a_{0}+a_{1}+\cdots \ldots+a_{k-1}\right)+A_{n}^{\prime}, n \geq k,
$$

It follows that $A=\lim _{n \rightarrow \infty} A_{n}$ exists (in the extended reals) if and only if:

$$
\begin{aligned}
& A^{\prime}=\lim _{n \rightarrow \infty} A_{n}^{\prime} \text { does, and in this case } \\
& A=\left(a_{0}+a_{1}+\cdots \ldots+a_{k-1}\right)+A^{\prime}[16] .
\end{aligned}
$$

\section{Lemma}

Suppose that for $n$ sufficiently large (that is, for $n \geq$ some integer $N$ ).

The terms of $\sum_{n=k}^{\infty} a_{n}$ satisfy some condition that implies convergence of an infinite series. Then $\sum_{n=k}^{\infty} a_{n}$ converges: Similarly, suppose that for $\mathrm{n}$ sufficiently large the terms $\sum_{n=k}^{\infty} a_{n}$ satisfy some condition that implies divergence of an infinite series: Then $\sum_{n=k}^{\infty} a_{n}$ diverges.

\section{E. Example}

Consider the alternating series test, which we will establish later as a special case of a more general test:

The series $\sum_{n=k}^{\infty} a_{n}$ converges if $(-1)^{n} a_{n}>0$,

$$
\left|a_{n+1}\right|<\left|a_{n}\right|, \text { and } \lim _{n \rightarrow \infty} a_{n}=0
$$

The terms of

$$
\sum_{n=1}^{\infty} \frac{16+(-2)^{n}}{n 2^{n}}
$$

Do not satisfy these conditions for all $n \geq 1$, but they do satisfy them for sufficiently large $n$. Hence, the series converges, by Lemma.

We will soon give several conditions concerning convergence of a series $\sum_{n=k}^{\infty} a_{n}$ with nonnegative terms. According to Lemma, these results apply to series that have at most finitely many negative terms, as long as $a_{n}$ is nonnegative and satisfies the conditions for $\mathrm{n}$ sufficiently large.

When we are interested only in whether $\sum_{n=k}^{\infty} a_{n}$ converges or diverges and not in its sum, we simply say " $\sum a_{n}$ converges" or " $\sum a_{n}$ diverges." Lemma (5-1) justifies this convention, subject to the understanding that $\sum a_{n}$ stands for $\sum_{n=k}^{\infty} a_{n}$, where $k$ is an integer such that $a_{n}$ is defined for $n \geq k$. (For example, $\sum \frac{1}{(n-6)^{2}}$ stands for $\sum_{n=k}^{\infty} \frac{1}{(n-6)^{2}}$ where $k \geq 7$.) We write $\sum a_{n}=\infty(-\infty)$ If $\sum a_{n}$ diverges to $\infty(-\infty)$ finally, let us agree that $\sum_{n=k}^{\infty} a_{n}$ and $\sum_{n=k-j}^{\infty} a_{n+j}$.

(Where we obtain the second expression by shifting the index in the first) both represent the same series. [3]

\section{F. Corollary}

If $\sum a_{n}$ converges; then $\lim _{n \rightarrow \infty} a_{n}=0$.It must be emphasized that Corollary (2.6) gives a necessary condition for convergence; that is $\sum a_{n}$ cannot converge unless $\lim _{n \rightarrow \infty} a_{n}=0$.The condition is not sufficient; $\sum a_{n}$ may diverge even if $\lim _{n \rightarrow \infty} a_{n}=0$. We will see examples below [13].

\section{G. Corollary}

If $\sum a_{n}$ converges; then for each $\epsilon>0$ there is an integer $K$ such that

$$
\left|\sum_{n=k}^{\infty} a_{n}\right|<\in \text { if } k \geq K
$$

That is,

$$
\lim _{k \rightarrow \infty} \sum_{n=k}^{\infty} a_{n}=0[10]
$$

\section{SERIES OF NonNEGATIVE TERMS}

The theory of series $\sum a_{n}$ with terms that are nonnegative for sufficiently large $n$ is simpler than the general theory, since such a series either converges to a finite limit or diverges to $\infty$, as the next theorem shows [8].

\section{A. Example}

Since $\frac{r^{n}}{n}<r^{\mathrm{n}}, n \geq 1$, and $\sum r^{n}<\infty$ if $0<r<1$, the series $\sum \frac{r^{n}}{n}$, converges if $0<r<1$, by the comparison test. Comparing these two series is inconclusive if $r>1$.

Since it does not help to know that the terms of $\sum \frac{r^{n}}{n}$ are smaller than those of the divergent series $\sum r^{n}$. If $r<0$, the comparison test does not apply since the series then have infinitely many negative terms.

\section{B. Example}

Since $r^{n}<n r^{n}$ and $\sum r^{n}$ if $r \geq 1$, the comparison test implies that $\sum n r^{n}=\infty$ if $r \geq 1$. Comparing these two series is inconclusive if $0<r<1$, since it does not help to know that the terms of $\sum n r^{n}$ are larger than those of the convergent series $\sum r^{n}$.

The comparison test is useful if we have a collection of series with nonnegative terms and known convergence properties. We will now use the comparison test to build such a collection [5].

\section{Corollary}

Suppose that $a_{n} \geq 0$ and $b_{n}>0$ for $n \geq k$, and $\lim _{n \rightarrow \infty} \frac{a_{n}}{b_{n}}=$ $L$.

Where $0<L<\infty$. Then $\sum a_{n}$ and $\sum b_{n}$ converge or diverge together.[14] 


\section{POWER SERIES}

A series having the form

$$
a_{0}+a_{1} x+a_{2} x^{2}+\cdots=\sum_{n=0}^{\infty} a_{n} x^{n}
$$

where $a_{0}, a_{1}, a_{2}, \ldots$ are constants, is called a power series in $x$.Itis often convenient to abbreviate the series (3) as $\sum a_{n} x^{n}$.

In general, a power series converges for $|x|<R$ and diverges for $|x|>R$, where the constant $R$ is called the radius of convergence of the series. For $|x|=\mathrm{R}$, the series may or may not converge.

The interval $|x|<R$ or $-R<x<R$, with possible inclusion of endpoints, is called the interval of convergence of the series. Although the ratio test is often successful in obtaining this interval, it may fail and in such cases, other tests may be used [9].

The two special cases $R=0$ and $R=\infty$ can arise. In the first case the series converges only for $x=0$ in the second case it converges for all $x$, sometimes written $-\infty<x<\infty$.

When we speak about a convergent power series, we assume, unless otherwise indicated, that $R>0$. Similar remarks hold for a power series of the form (3), where $x$ is replaced by $(x-a)[12]$.

\section{A. Example}

Test for convergence:

$$
1+2 r+r^{2}+2 r^{3}+r^{4}+2 r^{5}+\cdots
$$

where

$$
\text { (a) } r=\frac{2}{3} \text {, (b) } r=-\frac{2}{3} \text {, (c) } r=\frac{4}{3} \text {. }
$$

Here the ratio test is inapplicable, since $\left|\frac{u_{n+1}}{u_{n}}\right|=2|r|$ or $\frac{1}{2}|r|$ depending on whether $\mathrm{n}$ is odd or even However, using the nth root test, we have:

$$
\sqrt[n]{\left|u_{n}\right|}=\left\{\begin{array}{c}
\sqrt[n]{2\left|r^{n}\right|}=\sqrt[n]{2}|r| \text { if } n \text { is odd } \\
\sqrt[n]{\left|r^{n}\right|}=|r| \text { if } n \text { is even }
\end{array}\right.
$$

Then $\lim _{n \rightarrow \infty} \sqrt[n]{\left|u_{n}\right|}=|r|\left(\right.$ since $\left.\lim _{n \rightarrow \infty} 2^{\frac{1}{n}}=1\right)$.

Thus, if $|r|<1$ the series converges, and if $|r|>1$ the series diverges. Hence, the series converges for cases (a) and (b) and diverges in case (c) [15].

\section{B. Expansion of Functions in Power Series}

Here we get at the heart of the use of infinite series in analysis.

Functions are represented through them. Certain forms bear the names of mathematicians of the eighteenth and early nineteenth century who did so much to develop these ideas.

A simple way (and one often used to gain information in mathematics) to explore series representation of functions is to assume such a representation exists and then discover the details. of course, whatever is found must be confirmed in a rigorous manner. Therefore, assume:

$$
\begin{gathered}
f(x)=A_{0}+A_{1}(x-c)+A_{2}(x-c)^{2}+\cdots+A_{n}(x-2)^{n} \\
+\cdots
\end{gathered}
$$

Notice that the coefficients $A_{n}$ can be identified with derivatives of $f$. In particular:

$$
\begin{gathered}
A_{0}=f(c), A_{1}=f^{\prime}(c), A_{2}=\frac{1}{2 !} f^{\prime \prime}(c), \ldots, A_{n} \\
=\frac{1}{n !} f^{n}(c), \ldots
\end{gathered}
$$

This suggests that a series representation of $f$ is:

$$
\begin{aligned}
f(x)=f(c)+ & f^{\prime}(c)(x-c)+\frac{1}{2 !} f^{\prime \prime}(c)(x-c)^{2}+\cdots \\
& +\frac{1}{n !} f^{n}(c)(x-c)^{n}+\cdots
\end{aligned}
$$

A first step in formalizing series representation of a function, for which the first $\mathrm{n}$ derivatives exist, is accomplished by introducing Taylor polynomials of the function.

$$
\begin{aligned}
p_{0}(x) & =f(c), p_{1}(x)=f(c)+f^{\prime}(c)(x-c), \\
p_{2}(x) & =f(c)+f^{\prime}(c)(x-c)+\frac{1}{2 !} f^{\prime \prime}(c)(x-c)^{2}, \\
p_{n}(x) & =f(c)+f^{\prime}(c)(x-c)+\frac{1}{2 !} f^{\prime \prime}(c)(x-c)^{2}+\cdots+ \\
\frac{1}{n !} f^{n}(c)(x-c)^{n} . & \text { (4) [7]. }
\end{aligned}
$$

\section{EULER's SUMmATION FORMULA AND ASYMPTOTIC EXPANSIONS}

\section{A. The Summation Formula}

The range of action of all the summation processes with which we became acquainted. It is only when the terms $a_{n}$ of $\sum a_{n}$, the divergent series under consideration, do not increase too rapidly as $n$ increases that we can sum the series. Thus, in the case of the $B$-process, it is necessary that $\sum \frac{a_{n}}{n !} x^{n}$ should be convergent everywhere, i.e., that $\sqrt[n]{\frac{\left|a_{n}\right|}{n !}}$ or $\frac{1}{n} \sqrt[n]{\left|a_{n}\right|}$ should tend to zero. Hence the $B$-process cannot be used e. g. for the series:

$$
\begin{gathered}
\sum_{n=0}^{\infty}(-1)^{n} n !=1-1 !+2 !-3 !+4 !-5 !+\cdots+(-1)^{n} n ! \\
+\cdots
\end{gathered}
$$

Series like this one, and even more rapidly divergent series, occurred, however, in early investigations of the most varied kind. In order to deal conclusively with them by the methods used hitherto, we should have to introduce still more powerful processes, such as the $B_{r}$-process. However, no essential results have been obtained in this way.

At a fairly early stage in the development of the subject other methods were indicated, which in certain cases lead more conveniently to results useful both in theory and in practice. In the case of the numerical evaluation of the sum of an alternating series $\sum(-1)^{n} a_{n}$,

in which the $a_{n}$ 's constitute a positive monotone null sequence, we observed that the remainder $r_{n}$, always has the same sign as the first term neglected, and, moreover, that it is less than this term in absolute value [2]. 
Thus, in the calculation of the partial sums we need only continue until the terms have decreased down to the required degree of accuracy. A somewhat similar state of affairs exists in the case of the series:

$$
e^{-x}=1-x+\frac{x^{2}}{2}-\frac{x^{3}}{3}+\cdots+(-1)^{n} \frac{x^{n}}{n !}+\cdots, x>0
$$

Since the terms $\frac{x^{n}}{n !}$ likewise decrease monotone when $n>$ $x$. we can therefore write

$$
\begin{aligned}
e^{-x}=1-x+\frac{x^{2}}{2} & -\frac{x^{3}}{3}+\cdots+(-1)^{n} \frac{x^{n}}{n !} \\
& +(-1)^{n+1} \vartheta \frac{(n+1) !}{x^{(n+1)}}
\end{aligned}
$$

For every $n>x$, where $\vartheta$ of stands for a value between 0 and 1 , depending on $x$ and $n$, but is otherwise undetermined. It is impossible in practice, however, actually to calculate $e^{-x}$ from this formula when $x$ is large, for e.g. when $x=$ 1000 , the thousandth term is equal to $\frac{10^{3000}}{1000 !}$. As 1000 ! is a number with 2568 digits, the term under consideration is greater than $10^{431}$, so that the evaluation of the sum of the series cannot be carried out in practice. From the theoretical point of view, on the other hand, the series fulfills all requirements, since its terms, which (for large values of $x$ ) at first increase very rapidly, never the fewer ends by decreasing to zero, and that for every value of $x$. Hence any degree of accuracy whatever can be obtained in theory.

The circumstances are exactly the reverse, if we know that the value of a function $f(x)$ is represented by the formula:

$$
\begin{aligned}
& f(x)=1-\frac{1 !}{x}+\frac{2 !}{x^{2}}-\frac{3 !}{x^{3}}+\cdots+(-1)^{n} \frac{n !}{x^{n}}+ \\
& (-1)^{n+1} \frac{(n+1) !}{x^{(n+1)}},
\end{aligned}
$$

for every $n$. the series $\sum(-1)^{n} \frac{n !}{x^{n}}$, whose partial sums appear in this formula, diverges for every $x$ : but in contrast to nearly all the divergent series met with in the last chapter, the terms of the series (for large values of $x$ ) at first decrease very rapidly - the series at first behaves like a convergent one and it is only later on that they increase rapidly and without limit. Hence, we can calculate e. g. $f(1000)$ to about ten decimal places with great case; we have only to find an $\mathrm{n}$ for which $\frac{(n+1) !}{1000^{n+1}}<\frac{1}{2} 10^{-10}$. As this is true even for $n=3$, the value sought is given by:

$$
1-\frac{1}{10^{3}}+\frac{2}{10^{6}}-\frac{6}{10^{9}}
$$

to the desired degree of accuracy. Thus, it happens here that an expansion in powers, which takes the form of an infinite series which is divergent everywhere and very rapidly, so, nevertheless yields useful numerical results, because it appears along with its remainder. We are not in a position, however, - not even in theory - to obtain any degree of accuracy what- ever in the evaluation of $f(x)$, since $f(x)$ is given by its expansion only with an error of the order of one of the terms of the series. The degree of accuracy therefore cannot be lowered below the value of the least term of the series. (A least term certainly exists, seeing that the terms finally increase) As the example shows, however, in suitable circumstances all practical requirements may be satisfied.

Series of the type described were produced for the first time by Euler's summation formula, which we shall now consider more closely [2].

If the terms $a_{0}, a_{1}, a_{2}, \ldots a_{n}, \ldots$ of a series are the values of a function $f(x)$ for $x=0,1,2, \ldots, n, \ldots$, we have already proved by the integral test that in certain circumstances there is a relation between the partial sums $s_{n}=a_{0}+a_{1}+a_{2}+$ $\cdots+a_{n}$ and the integrals:

$$
J_{n}=\int_{0}^{n} f(x) d x \text {. }
$$

Euler's summation formula throws further light on this relation. If $f(x)$ possesses a continuous differential coefficient in $0 \leq x \leq n$, then, for:

$$
\begin{aligned}
& v=0,1, \ldots, n-1, \\
& \begin{aligned}
\int_{v}^{v+1}\left(x-v-\frac{1}{2}\right) & f^{\prime}(x) d x \\
& =\left(\left(x-v-\frac{1}{2}\right) f(x)\right)_{v}^{v+1} \\
& -\int_{v}^{v+1} f(x) d x
\end{aligned}
\end{aligned}
$$

Now, for each of the values $v$, we can put $v=(x)$ in the integrand on the left, at least for $v \leq x<v+1$. Since, however, the one value $x=v+1$ does not matter, we get:

$$
\begin{aligned}
& \frac{1}{2}\left(f_{v}+f_{v+1}\right)=\int_{v}^{v+1} f(x) d x+\int_{v}^{v+1}(x-[x]- \\
& \left.\frac{1}{2}\right) f^{\prime}(x) d x .
\end{aligned}
$$

(To simplify the writing, we denote by $f_{v}$ and $f_{v}^{(k)}$ respectively the values of $f(x)$ and of its derivative $f^{(k)}(x)$ for integral values $x=v$ ) Adding these relations for the relevant values of $\mathrm{v}$, and adjoining the term $\mathrm{s}\left(f_{0}+f_{n}\right)$, we finally obtain the formula.

$$
\begin{aligned}
& f_{0}+f_{1}+\cdots+f_{n}=\int_{0}^{n} f(x) d x+\frac{1}{2}\left(f_{0}+f_{n}\right)+\int_{0}^{n}(x- \\
& \left.[x]-\frac{1}{2}\right) f^{\prime}(x) d x(5)
\end{aligned}
$$

This in fact is Euler's summation formula in its simplest form. It gives a closed expression for the difference between the sums $f_{0}+f_{1}+\cdots+f_{n}$ and the corresponding integral $\int_{0}^{n} f(x) d x$.

We denote the function which appears in the last integrand by $P_{1}(x)$ :

$$
P_{1}(x)=x-[x]-\frac{1}{2}
$$

This is essentially the same function as the one which we met with in one of the first examples of Fourier expansions.

It is periodic, with period 1 , and for every non-integral value of $x$ we have

$$
P_{1}(x)=-\sum_{n=1}^{\infty} \frac{\sin 2 n \pi x}{n \pi} .
$$


A simple example to begin with will illustrate the importance of this formula. If $f(x)=\frac{1}{1+x}$, we obtain, by replacing $n$ by $n-1$,

$$
1+\frac{1}{2}+\cdots+\frac{1}{n}=\log n+\frac{1}{2}+\frac{1}{2 n}-\int_{1}^{n} \frac{p_{1}(x)}{x^{2}} d x .
$$

We may substitute the latter integral for $\int_{0}^{n-1} \frac{P_{1}(x)}{(1+x)^{2}}$, since $P_{1}(x+1)=P_{1}(x)$.

As pi $P_{1}(x)$, is bounded in $x>1$, the integral obviously converges when $n \rightarrow \infty$, and we find that

$$
\lim _{n \rightarrow \infty}\left(1+\frac{1}{2}+\cdots+\frac{1}{n}-\log n\right)=c=\frac{1}{2}-\int_{1}^{\infty} \frac{p_{1}(x)}{x^{2}} d x
$$

We already know that this limit exists, so we have a new proof of this fact, in addition to we have an expression in the form of an integral for Euler's constant $c$, by means of which we can evaluate the constant numerically. From the formula (6) i.e.

$$
\begin{aligned}
& f_{0}+f_{1}+\cdots+f_{n}=\int_{0}^{n} f(x) d x+\frac{1}{2}\left(f_{0}+f_{n}\right)+ \\
& \int_{0}^{n} P_{1}(x) f^{\prime}(x) d x
\end{aligned}
$$

Integration by parts leads to more advantageous representations. In order to be in a position to carry it out, we must first assume that $f(x)$ has continuous derivatives of all the orders which occur in what follows; then we have to select an indefinite integral of $P_{1}(x)$, and an integral of the latter, and so on. By suitable choice of the constants of integration the further calculations are greatly simplified. We shall follow wringers and set:

$$
P_{2}(x)=+\sum_{n=1}^{\infty} \frac{2 \cos 2 n \pi x}{(2 n \pi)^{3}} .
$$

Then $P_{2}^{\prime}(x)=P_{1}(x)$, for every non-integral value of $x$, and $P_{2}(0)=\frac{1}{2 \pi^{2}} \sum_{n=1}^{\infty} \frac{1}{n^{2}}=\frac{1}{12}$. Moreover, $P_{2}(x)$ is continuous throughout and has the period 1 . We now proceed to set:

$$
P_{3}(x)=+\sum_{n=1}^{\infty} \frac{2 \sin 2 n \pi x}{(2 n \pi)^{3}}
$$

Whence we have $P^{\prime}{ }_{3}(x)=P_{2}(x)$, for every value of $x$, $P_{3}(0)=0$, and in general:

$$
\left\{\begin{array}{l}
P_{2 \lambda}=(-1)^{\lambda-1} \sum_{n=1}^{\infty} \frac{2 \cos 2 n \pi x}{(2 n \pi)^{2 \lambda}}, \\
P_{2 \lambda+1}=(-1)^{\lambda-1} \sum_{n=1}^{\infty} \frac{2 \sin 2 n \pi x}{(2 n \pi)^{2 \lambda+1}}
\end{array}\right.
$$

Then, for $\lambda=1,2,3, \ldots$, all these functions are throughout continuous and continuously differentiable, and have the period 1; and we have:

$$
\left\{\begin{array}{c}
P^{\prime}{ }_{k+1}(x)=P_{k}(x) \\
P_{2 \lambda}(0)=(-1)^{\lambda-1} \sum_{n=1}^{\infty} \frac{2}{(2 n \pi)^{2 \lambda}}=\frac{B 2 \lambda}{(2 \lambda)^{2 n}}, P_{2 \lambda+1}(0)=0
\end{array}\right.
$$

for $k, \lambda=1,2, \ldots\left(\sum_{n=1}^{\infty} \frac{1}{n^{2 p}}=(-1)^{p-1} \frac{B_{2 p}(2 \pi)^{2 p}}{2(2 p) !}(p\right.$ fixed $\left.)\right)$

As is immediately obvious from the proof.

In the interval $0 \leq x \leq 1$ and for $k \geq 2$, the functions $P_{k}(x)$ are rational integral functions. Besides the fact that $P_{1}(x)=x-\frac{1}{2}$ in $0<x<1$,

we have, in $0 \leq x \leq 1$,

$$
\begin{gathered}
P_{2}(x)=\frac{x^{2}}{2}-\frac{x}{2}+\frac{1}{12}=\frac{x^{2}}{2 !}+\frac{B_{1}}{1 !} \frac{x}{1 !}+\frac{B_{2}}{2 !}, \\
P_{3}(x)=\frac{x^{3}}{6}-\frac{x^{2}}{4}+\frac{x}{12}=\frac{x^{3}}{3 !}+\frac{B_{1}}{1 !} \frac{x^{2}}{2 !}+\frac{B_{2}}{2 !} \frac{x}{1 !} \\
P_{4}(x)=\frac{x^{4}}{24}-\frac{x^{3}}{12}+\frac{x^{2}}{24}-\frac{1}{720}=\frac{x^{4}}{4 !}+\frac{B_{1}}{1 !} \frac{x^{3}}{3 !}+\frac{B_{2}}{2 !} \frac{x^{2}}{2 !}+\frac{B_{4}}{4 !}
\end{gathered}
$$

Hence in general, as may immediately be established by induction:

$$
\begin{gathered}
P_{k}(x)=\frac{x^{k}}{k !}+\frac{B_{1}}{1 !} \frac{x^{k-1}}{(k-1) !}+\frac{B_{2}}{2 !} \frac{x^{(k-2)}}{(k-2) !}+\cdots+\frac{B_{k}}{k !}= \\
\frac{1}{k !}\left(\begin{array}{l}
k \\
0
\end{array}\right) x^{k}+\left(\begin{array}{l}
k \\
1
\end{array}\right) B_{1} x^{k-1}+\left(\begin{array}{l}
k \\
2
\end{array}\right) B_{2} x^{k-2}+\cdots \\
\left.+\left(\begin{array}{c}
k \\
k-1
\end{array}\right) B_{k-1}(x)+\left(\begin{array}{l}
k \\
k
\end{array}\right) B_{k}\right)
\end{gathered}
$$

Or

(c) $P_{K}(x)=\frac{1}{k !}(x+B)^{k}$

If we employ the symbolic notation already used. These are the so-called Bernoulli's polynomials, which play an important part in many investigations. We shall meet with some of their important properties directly.

First of all, however, we shall improve the formula by means of these polynomials. Integration by parts gives:

$$
\begin{gathered}
\int_{0}^{n} P_{1}(x) f^{\prime}(x) d x=\left(P_{2} f^{\prime}\right)_{0}^{n}-\int_{0}^{n} P_{2} f^{\prime \prime} d x=\frac{B_{2}}{2 !}\left(f^{\prime}{ }_{n}-\right. \\
\left.f^{\prime}{ }_{0}\right)-\left(P_{3} f^{\prime \prime}\right)_{0}^{n}+\int_{0}^{n} P_{3} f^{\prime \prime \prime} d x \\
=\frac{B_{2}}{2 !}\left(f^{\prime}{ }_{n}-f^{\prime}{ }_{0}\right)+\int_{0}^{n} P_{3} f^{\prime \prime \prime} d x
\end{gathered}
$$

And, generally,

$$
\begin{aligned}
& \int_{0}^{n} P_{2 \lambda-1} f^{(2 \lambda-1)} d x \\
= & \frac{B_{2 \lambda}}{(2 \lambda) !}\left(f_{n}^{(2 \lambda-1)}-f_{0}^{(2 \lambda-1)}\right) \\
+ & \int_{0}^{n} P_{2 \lambda+1} f^{(2 \lambda+1)} d x
\end{aligned}
$$

for $\lambda \geq 1$. Hence, for every $k \geq 0$, provided only that the derivatives of $f(x)$ involved exist and are continuous, we can write:

$$
\begin{gathered}
f_{0}+f_{1}+\cdots+f_{n}=\int_{0}^{n} f(x) d x+\frac{1}{2}\left(f_{n}+f_{0}\right)+\frac{B_{2}}{2 !}\left(f_{n}^{\prime}-\right. \\
\left.f_{0}^{\prime}\right)+\frac{B_{4}}{4 !}\left(f_{n}^{\prime \prime \prime}-f_{0}^{\prime \prime \prime}\right)+\cdots+\frac{B_{2 k}}{(2 k) !}\left(f_{n}^{(2 k-1)}-f_{0}^{(2 k-1)}\right)+R_{k}
\end{gathered}
$$


Where we put:

$$
R_{k}=\int_{0}^{n} P_{2 k+1}(x) f^{(2 k+1)}(x) d x
$$

This is Euler's summation formula [2].

Some Applications:

1. It is obvious that the most favorable results are obtained when. the higher derivatives of $f(x)$ are very small, and especially when they vanish. We therefore first choose $f(x)=x^{P}$, where $p$ is an integer $\geq 1$, and we have:

2.

$1^{P}+2^{P}+3^{P}+\cdots+n^{P}=\int_{0}^{n} x^{P} d x+\frac{1}{2} n^{P}+$ $\frac{B_{2}}{2 !} P n^{P-1}+\cdots$

Here the series on the right hand side is to be broken off at the last positive power of $n$, for $\left(f_{n}^{(k)}-f_{0}^{(k)}\right)$ vanishes not only when $f^{(k)}(x)=0$, but also when $f^{(k)}(x)$ is identically equal to a non-vanishing constant.

Thus, by transferring $n^{P}$ to the right hand side we have:

$$
1^{P}+2^{P}+\cdots+(n-1)^{P}=\frac{1}{P+1}\left(n^{P+1}+\left(\begin{array}{c}
P+1 \\
1
\end{array}\right) B_{1} n^{P}+\right.
$$$$
\left.\left(\begin{array}{c}
P+1 \\
2
\end{array}\right) B_{2} n^{P-1}+\cdots\right)
$$

or - since there is no constant term appearing inside the brackets on the right hand side:

$$
1^{P}+2^{P}+\cdots+(n-1)^{P}=\frac{1}{P+1}\left((n+B)^{P+1}-B^{P+1}\right)
$$

2. The sums dealt with the above can be obtained in quite a different way. If we

imagine that each term of the sum:

$$
1+e^{t}+e^{2 t}+\cdots+e^{(n-1) t}
$$

is expanded in powers of $t$, the coefficient of $\frac{t^{P}}{P !}$ is obviously

$$
1^{P}+2^{P}+\cdots+(n-1)^{P}
$$

On the other hand, if we use symbolic notation, the first sum is equal to:

$$
\frac{e^{n t}-1}{e^{t}-1}=\frac{e^{n t}-1}{t} e^{B t}=\frac{e^{(n+B) t}-e^{B t}}{t} .
$$

Hence, we immediately obtain the expression

$$
\frac{1}{P+1}\left((n+B)^{P+1}-B^{P+1}\right)
$$

For the coefficient of $\frac{t^{P}}{P !}$.

3. If we put $f(x)=e^{a x}, n=1$, we obtain:

4.

$$
\begin{aligned}
\frac{1}{2}\left(e^{\alpha}+1\right)= & \frac{e^{\alpha}-1}{\alpha}+\sum_{v=1}^{k} \frac{B_{2 v}}{(2 v) !} \alpha^{2 v-1}\left(e^{\alpha}-1\right) \\
& +\alpha^{2 k+1} \int_{0}^{1} P_{2 k+1}(x) e^{\alpha x} d x
\end{aligned}
$$

Or

$$
\begin{gathered}
\frac{\alpha}{e^{\alpha}-1}=1-\frac{\alpha}{2}+\sum_{v=1}^{\infty} \frac{B_{2 v}}{(2 v) !} \alpha^{2 v}+ \\
\frac{\alpha^{2 k+2}}{e^{\alpha}-1} \int_{0}^{1} P_{2 k+1}(x) e^{\alpha x} d x .
\end{gathered}
$$

Since we can immediately prove, that the remainder tends to zero in this case, provided only that $|\alpha|<2 \pi$, we have, for these values of $\alpha$,

$$
\frac{\alpha}{e^{\alpha}-1}=1-\frac{\alpha}{2}+\sum_{v=1}^{\infty} \frac{B_{2 v}}{(2 v) !} \alpha^{2 v}=\sum_{\lambda=1}^{\infty} \frac{B_{\lambda}}{\lambda !} \alpha^{\lambda}
$$

Similarly, by putting $f(x)=\cos \alpha x, n=1$, we obtain the expansion for $\frac{\alpha}{2} \cot \frac{\alpha}{2}$.

5. If we put $f(x)=\frac{1}{1+x}$, we have, by replacing $n$ by $(n-1)$,

6.

$$
\begin{aligned}
& 1+\frac{1}{2}+\cdots+\frac{1}{n}=\log n+\frac{1}{2}+\frac{1}{2 n}+\frac{B_{2}}{2}\left(1-\frac{1}{n^{2}}\right) \\
& +\frac{B_{4}}{4}\left(1-\frac{1}{n^{4}}\right)+\text {. } \\
& . .+\frac{B_{2 k}}{2 k}\left(1-\frac{1}{n^{2 k}}\right)-(2 k+1) ! \int_{1}^{n} \frac{P_{2 k+1}(x)}{x^{2 k+2}} d x \text {. }
\end{aligned}
$$

Since here we may let $n \rightarrow \infty$, we obtain the following refined expression for Euler's constant:

$$
c=\frac{1}{2}+\frac{B_{2}}{2}+\frac{B_{4}}{4}+\cdots+\frac{B_{2 k}}{2 k}-(2 k+1) ! \int_{1}^{\infty} \frac{P_{2 k+1}(x)}{x^{2 k+2}} d x .
$$

In this case the remainder certainly does not decrease to 0 as $k$ increases; and the series $\sum \frac{B_{2 k}}{2 k}$ diverges rapidly, so rapidly that even the corresponding power series $\sum \frac{B_{2 k}}{2 k} x^{2 k}$ diverges everywhere; for, by

$$
\begin{aligned}
& \sum_{n=1}^{\infty} \frac{1}{n^{2 p}}=(-1)^{p-1} \frac{B_{2 p}(2 \pi)^{2 p}}{2(2 p) !}(p \text { fixed }), \\
& \left|B_{2 k}\right|=\frac{2(2 k) !}{(2 \pi)^{2 k}} \eta \text { where } 1<\eta<2 .
\end{aligned}
$$

Nevertheless, we can evaluate $c$ very accurately by means of the above expression. If we take e. g. $k=3$, we have, in the first instance.

(a) $C=\frac{1}{2}+\frac{1}{12}-\frac{1}{120}+\frac{1}{252}-7 ! \int_{1}^{\infty} \frac{P_{7}(x)}{x^{3}} d x$.

If we take only the part of the integral from $x=1$ to $x=$ 4 , the absolute value of the error is:

$$
\leq 7 ! \frac{4}{(2 \pi)^{7}} \int_{4}^{\infty} \frac{d x}{x^{3}}
$$

Hence,

$$
c=\frac{1459}{2520}-7 ! \int_{1}^{4} \frac{P_{7}(x)}{x^{8}} d x+\frac{\eta}{10^{6}}
$$

where

$|\eta|<1$. 
The required evaluation of the integral is also given by the first formula written down, for $n=4$, namely:

$-7 ! \int_{1}^{4} \frac{P_{7}(x)}{x^{8}} d x=1+\frac{1}{2}+\frac{1}{3}+\frac{1}{4}-\log 4-\frac{1459}{2520}-\frac{1}{2.4}+$
$\frac{1}{12.4^{2}}-\frac{1}{120.4^{4}}+\frac{1}{252.4^{6}}$.

Hence,

$0.5772146<c<0.5772168$.

In this way we can easily obtain $c$ with much greater accuracy than before, and theoretically to any degree of accuracy whatever. The reason for this favorable state of affairs lies solely in the fact that we may regard the logarithms as known.

\section{RESULTS}

The paper dealt with the infinite series and some applications to Euler's summation, and we took the model study (Euler's summation) and we came to the results of which is access to the relationship through the Euler's summation and we focused on the physical link questioner to make the study as an application for Euler's summation so it can be the beginning of advanced study in concept of the infinite series and its applications to Euler's summation.

\section{ACKNOWLEDGMENT}

First of all, we thank God Almighty who succeeded in writing this research paper. And nice thanks to everyone who contributed to expressing their observations and criticisms until it appeared in this way.

\section{REFERENCES}

[1] E T. Whittaker, M, A- A course of Modern Analysis -London. -1902.

[2] DR. Konrad Knopp - Theory and Application of Infinite Series-Fourth German Edition, 1951.

[3] H.S. Carslaw - Introduction to the Theory of Fourier's Series and Integrals-Second Edition Llondon-1921.

[4] James. M. Hysiop -Infinite Series-New York-1959.

[5] James Nearing- Mathematical Tools for Physics- Copyright 2003.

[6] John Wiley \&Sons - Calculus Volume II -Second Edition -London copyright 1962.

[7] Malcolm R. Adams -Sequences and Series and Introduction to Mathematical Analysis 2007.

[8] Maria Predoi - Mathematical Analysis Vol. II Integral Calculus 2005.

[9] Michael Corral -Vector Calculus- Copyright 2008.

[10] Omar Hijab -Introduction to Calculus and Classical Analysis-Second Edition-2007.

[11] R. B. Dingle- Asymptotic Expansions Their Derivation and Interpretation- Acndemic Press Inc-London- Copyright 1973.

[12] Robert Wrede, Ph. D.Murrayr. Spiegel, Ph.D. -Theory and Problems of Advanced Calculus-Second Edition-Schaum's Outline Series-New York-Copyright 2002, 1963 by The McGraw-Hill Companies.

[13] Sean- Introduction to Methods of Applied Mathematics-AntiCopyright 1995-2001- Mauch Publishing Company-January 24, 2004.

[14] Sever Angel Popescu - Mathematical Analysis Integral Calculus Bucharest-February, 2011.

[15] Thaddeus H. Black- Derivations of Applied Mathematics-Copyright 1983-2012.

[16] T.J. PA. Bromwh'h. M.A- An Introduction to the Theory of Infinite Series - London 1908.

[17] William F. Trench- Introduction to Real Analysis -Texas, Free Edition- 24 December 2013. 